\title{
Instrument for the measurement of hysteresis loops of magnetotactic bacteria and other systems containing submicron magnetic particles
}

\author{
Hendrik de Waard \\ Nuclear Solid State Physics Department, University of Groningen, The Netherlands \\ James Hilsinger and Richard B. Frankel ${ }^{\text {a) }}$ \\ Department of Physics, California Polytechnic State University, San Luis Obispo, California 93407
}

(Received 13 June 2000; accepted for publication 5 February 2001)

\begin{abstract}
An electronic control system for the measurement of hysteresis curves of microscopically observed magnetic structures such as chains of magnetosomes in magnetotactic bacteria suspended or swimming in water is described. Using continuous magnetic fields generated by four coils for guidance or orientation of the bacteria or other magnetic structures, and pulsed magnetic fields in two additional coils for changing the degree of magnetization in small steps, hysteresis curves can be traversed. The circuits described can be constructed with readily available components. The guiding- and pulsed-field coils can be fashioned in any standard machine shop. The typical sensitivity of the system is better than $10^{-12} \mathrm{ergs} / \mathrm{G}$, which makes a wide range of bacteria accessible to quantitative measurement of their magnetosome chains. The electronic circuits as well as the coil systems are described in detail. (C) 2001 American Institute of Physics.
\end{abstract}

[DOI: $10.1063 / 1.1361079]$

\section{INTRODUCTION}

After the discovery by Blakemore ${ }^{1}$ of magnetotactic bacteria in 1975, many different types of these bacteria have been found in marine and freshwater environments around the world. ${ }^{2-4}$ Magnetotactic bacteria swim in or against the direction of an external magnetic field. Their orientation along magnetic-field lines is due to intracytoplasmic "magnetosomes," i.e., membrane-enclosed magnetic iron mineral particles, ranging in size from about $40-100 \mathrm{~nm}$. In most magnetotactic bacteria the iron mineral is magnetite $\left(\mathrm{Fe}_{3} \mathrm{O}_{4}\right)$, but in some marine organisms the mineral is greigite $\left(\mathrm{Fe}_{3} \mathrm{~S}_{4}\right)$. The magnetosomes are usually arranged in one or more chains and comprise a permanent magnetic dipole of the order of $10^{-12} \mathrm{emu}(\mathrm{ergs} / \mathrm{G})$ that is fixed within the cell. ${ }^{5} \mathrm{~A}$ bacterium is oriented as it swims by the torque exerted by the external magnetic field (the geomagnetic field in natural environments) on its magnetic dipole. ${ }^{6}$ The instrument described here was designed to make hysteresis measurements on magnetosome chains in individual cells.

The hysteresis measurements are performed for individual bacteria suspended in water within a capillary of rectangular cross section and viewed with a microscope or recorded with a video camera (see Fig. 1). The method is essentially the same as that used previously by Knowles. ${ }^{7}$ Points on the hysteresis curve are obtained as follows:

(1) With a magnetic bacterium oriented in a dc external "guiding" field $\mathbf{B}_{\mathbf{G}}$ (up to about $10 \mathrm{G}$ ), a strong magnetic pulse $\mathbf{B}_{\mathbf{p}}$ (peak amplitude up to $800 \mathrm{G}$ and 1-3 ms duration) is applied in the direction of the guiding field (we will call this direction positive). This results in the maximum (satura-

${ }^{a)}$ Electronic mail: rfrankel@calpoly.edu tion) remanent magnetization in the bacterium's magnetosome chain.

(2) The bacterium is then subjected to increasing negative pulses $\mathbf{B}_{\mathbf{P}}$ (the pulse-field amplitude is generally increased in small steps to provide sufficient detail in the loop). When the pulse field has exceeded the coercive force of the magnetosome chain, the cell will be observed to rotate $180^{\circ}$ immediately following the pulse.

(3) After each pulse, the guiding field is reversed and the time required for rotation of the bacterium over a welldefined angle perpendicular to the rotation axis is determined. Alternatively, the dc guiding field can be rotated in the horizontal plane at fixed frequency. The amplitude of the rotating field is then decreased until the cell stops following the rotating field. Either of these measurements can be used to determine the remanent magnetization following the pulse, relative to the saturation remanent magnetization.

(4) This process is repeated for regularly increasing values of the pulse-field amplitude until saturation remanent magnetization in the negative direction is achieved. The first half of the hysteresis curve can now be drawn through the measured points. After this, the bacterium can be rotated $180^{\circ}$ by the guiding field and the process continued to measure the other half of the hysteresis curve.

Recently, we published the results of hysteresis measurements for three different types of magnetotactic bacteria. ${ }^{8}$ In that paper, we also gave a more-detailed account of the method of measurement, including the influence of the viscosity of the liquid and viscous drag on the cell.

In view of the more general interest in the properties of acicular, submicron magnetic particles, ${ }^{7}$ as well as of other types of magnetic bacteria than those already described in Ref. 8, we give here a more-detailed description of the instrument. It is intended to help anyone interested in the con- 


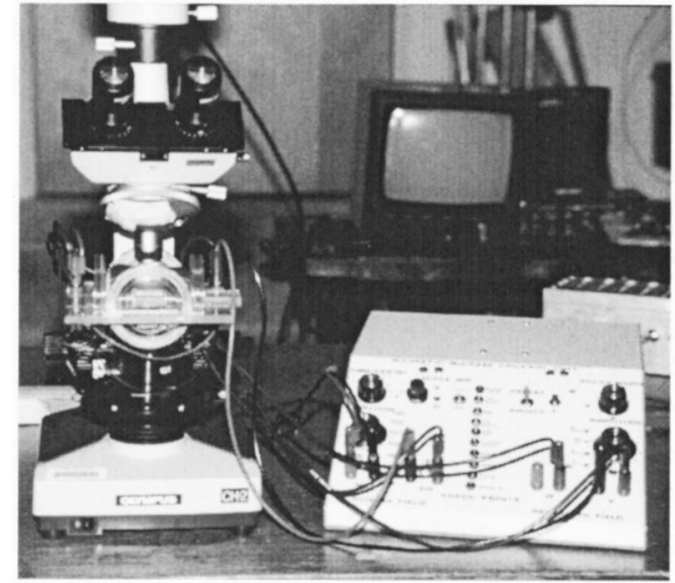

FIG. 1. Photograph of the magnetometer. Left: Olympus BH-2 microscope with video camera and coil system on microscope stage. Right: control panel for guiding magnetic bacteria and changing their magnetization.

struction of the magnetometer that has some experience in building electronic instruments. The instrument might also be used to distinguish magnetic from nonmagnetic bacteria in natural samples, ${ }^{9}$ or for light scattering by rotating submi- cron magnetic particles or rotating bacteria. ${ }^{10}$ It could be useful in experiments in which nonmagnetic mutants are generated from magnetic cells for genetic analysis, or in genetic engineering involving magnetic bacteria.

\section{GENERAL DESCRIPTION OF THE MAGNETOMETER}

A photograph of the magnetometer system is shown in Fig. 1. At the left is an Olympus BH-2 microscope in which the normal, metal, microscope stage has been replaced with a lucite stage holding a multiple magnetic coil system and a capillary containing the magnetic bacteria (or other samples). The capillary can be moved over a few $\mathrm{mm}$ in the northsouth (NS) and east-west (EW) directions so as to select the part of the specimen to be investigated. The sample can be viewed simultaneously through the standard binocular eyepieces and a mono-ocular connected to a TV camera. On the right is the control system for energizing the coils. A perspective rendering of the coil system is shown in Fig. 2(a). The functions of the controls are given in Fig. 2(b) and further defined in Secs. III A-III G (see, also, Table I).

The magnetometer comprises circular coils that generate horizontal magnetic fields in the plane of the microscope

(a)

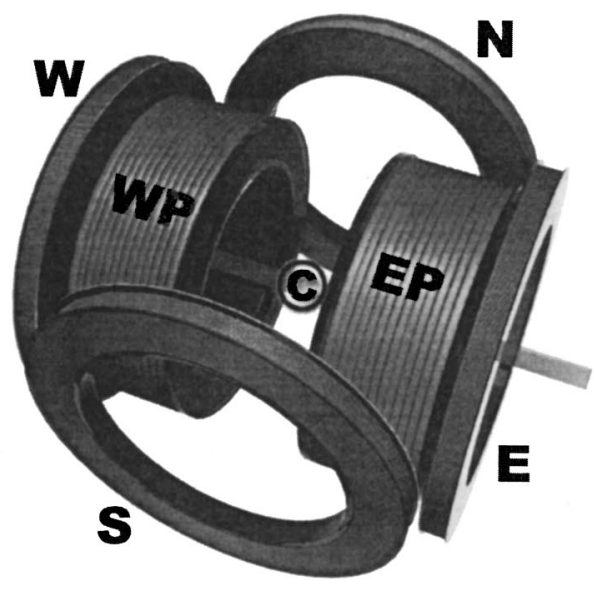

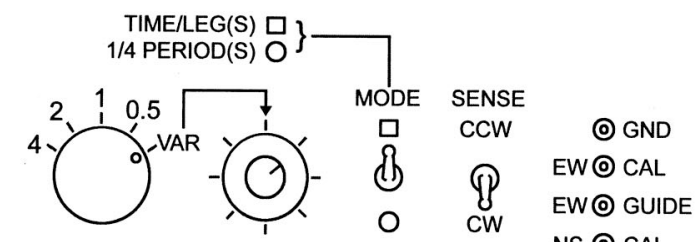

NS $\odot$ CAL

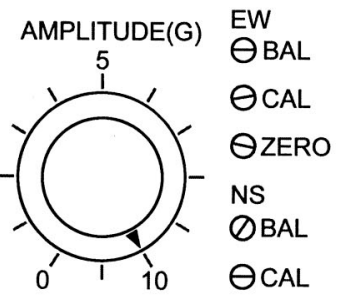

QZERO

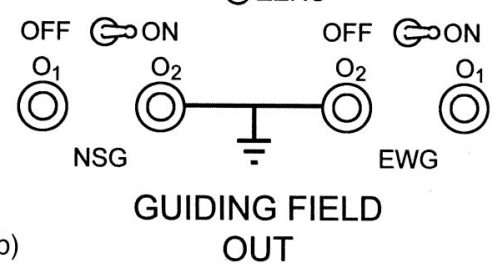

๑ LOGIC $W$ ๑ PULSE $W$ ○ ZERO

E ๑ PULSE

E $\odot$ ZERO

○ DVM

OUT
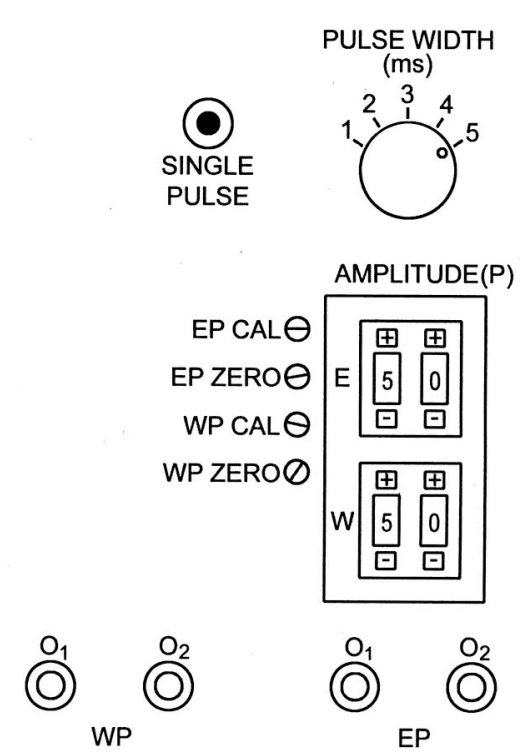

PULSE FIELDS

OUT
FIG. 2. (a) Coil system in perspective view. The four coils $\mathrm{N}, \mathrm{W}, \mathrm{E}$, and $\mathrm{S}$, placed in square, provide the guiding field that steers the swimming bacteria. The two coils EP and WP inside the square generate a pulse field that can influence the degree of magnetization of the bacteria held at the center. (b) Front panel of the control unit. Most of the switches, variable controls, and test points are discussed in the circuit descriptions (see Secs. III A-III G). 
TABLE I. Front-panel controls and connectors shown in Fig. 2(b), with definitions and connection to the circuits given in Figs. 3(b)-3(g). Note that when there are separate amplifiers, as for the NS and EW guiding fields, and for the EP and WP fields, the component designations in the circuits are the same for both channels.

\begin{tabular}{|c|c|}
\hline Controls and connectors & Circuit designations \\
\hline \multicolumn{2}{|l|}{ Left (top to bottom) } \\
\hline Time/leg(s) & Square mode: switch $R_{5}$ in Fig. 3(b) \\
\hline $1 / 4 \operatorname{period}(\mathrm{s})$ & Circular mode: switch $R_{3}$ in Fig. 3(c) \\
\hline Sense $\mathrm{CCW} / \mathrm{CW}$ & See Sec. III G \\
\hline Amplitude (G) & Guiding-field square/circle amplitude pot $R_{1}$ \\
\hline $\mathrm{ON}-\mathrm{OFF}$ & Output of Fig. 3(e) to NS or EW coils $\left(L_{1}, L_{2}\right)$ \\
\hline $\mathrm{O} 1, \mathrm{O} 2(2 \mathrm{X})$ & Outputs of Fig. 3(e) to NS or EW coils \\
\hline \multicolumn{2}{|l|}{ Right (top to bottom) } \\
\hline Single pulse & Push-button $S_{1}$ in Fig. 3(d) \\
\hline Pulse width & Switched resistor $R_{1}$ in Fig. 3(d) \\
\hline Amplitude (P) & Thumb wheel pots $R_{22}$ in Fig. 3(f) \\
\hline $\mathrm{O} 1, \mathrm{O} 1, \mathrm{O} 2,(2 \mathrm{X})$ & Connectors from Fig. 3(f) to EP and WP coils \\
\hline \multicolumn{2}{|l|}{ Middle (top to bottom) } \\
\hline \multicolumn{2}{|l|}{ Test connectors (in) } \\
\hline GND & Ground (chassis) \\
\hline EW CAL & $R_{10}$ in Fig. 3(e) (EW AMPL) \\
\hline EW GUIDE & TP1 in Fig. 3(e) (EW AMPL) \\
\hline NS CAL & $R_{10}$ in Fig. 3(e) (NS AMPL) \\
\hline NS GUIDE & TP1 in Fig. 3(e) (NS AMPL) \\
\hline \multicolumn{2}{|l|}{ Test connectors (out) } \\
\hline \multirow[t]{2}{*}{ LOGIC } & Square: Fig. 3(b) EWG, NSG \\
\hline & Circle: Fig. 3(c) EWG, NSG \\
\hline W PULSE & TP1 wrt GND Fig. 3(f) \\
\hline W ZERO & TP2 wrt GND Fig. 3(f) \\
\hline E PULSE & TP1 wrt GND Fig. 3(f) \\
\hline E ZERO & TP2 wrt GND Fig. 3(f) \\
\hline DVM & Digital voltmeter to GND Fig. 3(g) \\
\hline \multicolumn{2}{|l|}{ Trimpots } \\
\hline \multicolumn{2}{|l|}{ Left (top to bottom) } \\
\hline EW BAL & $R_{8}$ in Fig. 3(e) \\
\hline EW CAL & $R_{10}$ in Fig. 3(e) \\
\hline EW ZERO & $R_{9}$ in Fig. 3(e) \\
\hline NS ZERO & $R_{9}$ in Fig. 3(e) \\
\hline \multicolumn{2}{|l|}{ Right (top to bottom) } \\
\hline EP CAL & $R_{19}$ in Fig. 3(f) \\
\hline EP ZERO & $R_{18}$ in Fig. 3(f) \\
\hline WP CAL & $R_{19}$ in Fig. 3(f) \\
\hline WP ZERO & $R_{18}$ in Fig. 3(f) \\
\hline
\end{tabular}

stage, on which is placed the thin, flat capillary tube containing the bacteria in water. Two types of field are generated: a guiding magnetic field that steers the bacteria along prescribed paths through the liquid and a pulsed magnetic field that allows the magnetization of the bacteria to be changed in steps. Two pairs of flat coils, designated NS and EW generate the guiding field. Each of the four guiding field coils, N, $\mathrm{S}, \mathrm{E}$, and $\mathrm{W}$, consists of 150 turns of 0.5 -mm-diam, insulated, copper wire (coil resistance $2.5 \Omega$ ) wound on circular lucite forms $(6.7 \mathrm{~cm}$ o.d., $5.1 \mathrm{~cm}$ i.d., width $6 \mathrm{~mm})$. The coil planes are vertical and placed in a square so that the NS and EW magnetic-field axes cross each other in the middle $(C)$ of the horizontal plane, where a thin, flat capillary containing the sample is placed [Fig. 2(a)]. The NS and EW guidingfield coils are so connected in series that the magnetic fields generated by them add in the center.
By energizing the two pairs of coils sequentially, the bacteria can be made to swim in a square horizontal path. Nonmotile cells rotate in $90^{\circ}$ steps. For some measurements, a continuously rotating magnetic field is desired, ${ }^{7,9,10}$ which would cause the cells to swim in a circular path or cause nonmotile bacteria to rotate continuously about the center of their permanent magnetic dipole. For the rotating field, sinusoidal currents simultaneously energize the NS and EW coil pairs with a $90^{\circ}$ phase difference between them.

The horizontal pulsed magnetic field is generated by another pair of coils oriented parallel to each other and placed between the E and W guiding-field coils, closer to the capillary tube. The two pulsed-field coils [east pulsed (EP) and west-pulsed (WP)] [Fig. 2(a)], each consist of 600 turns of 0.5-mm-diam insulated copper wire (coil resistance $7.4 \Omega$ ) and are also circular $(5.5 \mathrm{~cm}$ o.d., $3.0 \mathrm{~cm}$ i.d., width $2.0 \mathrm{~cm})$. They are placed so that their inner flanges are $21.4 \mathrm{~mm}$ apart. The capillary is generally placed at $\mathrm{C}$, midway between the pulse-field coils on the common axes of all coils. Since the sample is usually small compared with the distance between the coils, the guiding and pulsed fields are quite homogeneous at the position of the sample. The pulse width can be varied from 1 to $5 \mathrm{~ms}$ in five steps, while the field amplitude can be varied in small steps from 0 to about \pm 800 G. Saturation magnetization of most magnetic bacteria is reached for a pulsed field greater than about $600 \mathrm{G}$. The maximum value of the pulsed field is determined after each pulse by a peakfield meter. The peak-measuring circuit is described in Sec. III F.

The pulsed field can also be used in conjunction with a dc guiding field. In this case, the EW guiding-field coil pair is connected to an external dc current source through a reversing switch and the NS guiding-field coil pair is switched off.

The magnetic fields are calibrated with the aid of a Hallprobe magnetometer that replaces the capillary at the center (C) of the coils. The pulsed-field amplitude is calibrated such that one digital step of the pulsed field corresponds to $10 \mathrm{G}$. The field $B_{P}$ midway between the coils and on their common axis is set to $70(0.5) \mathrm{G} / \mathrm{A}$ per coil. The voltage across $10 \Omega$ resistors placed in series with the coils is measured with an oscilloscope (E,W PULSE OUT). The simple relation between the field $B_{P}$ and the measured voltages $V_{P}(W)$ $=V_{P}(E)$ for the calibrated system is

$$
B_{P}(\mathrm{G})=14 \quad V_{P}(\mathrm{~V}) \text {. }
$$

The accuracy of this calibration, taking into account the existing calibration of a standard oscilloscope, is of the order of $\pm 2 \%$.

The heat dissipation in the coils is moderate. In the guiding-field coils at the highest current (about $2 \mathrm{~A} /$ coil) and with equal loading of the coils, the heat produced per coil is about $2 \mathrm{~W}$. However, at a constant maximum current the energized coil would overheat if the switching provided by the logic stops. The two pulse coils (EP and WP) will not be overloaded as long as the relative pulse width is less than $1 \%$ of the repetition rate. 

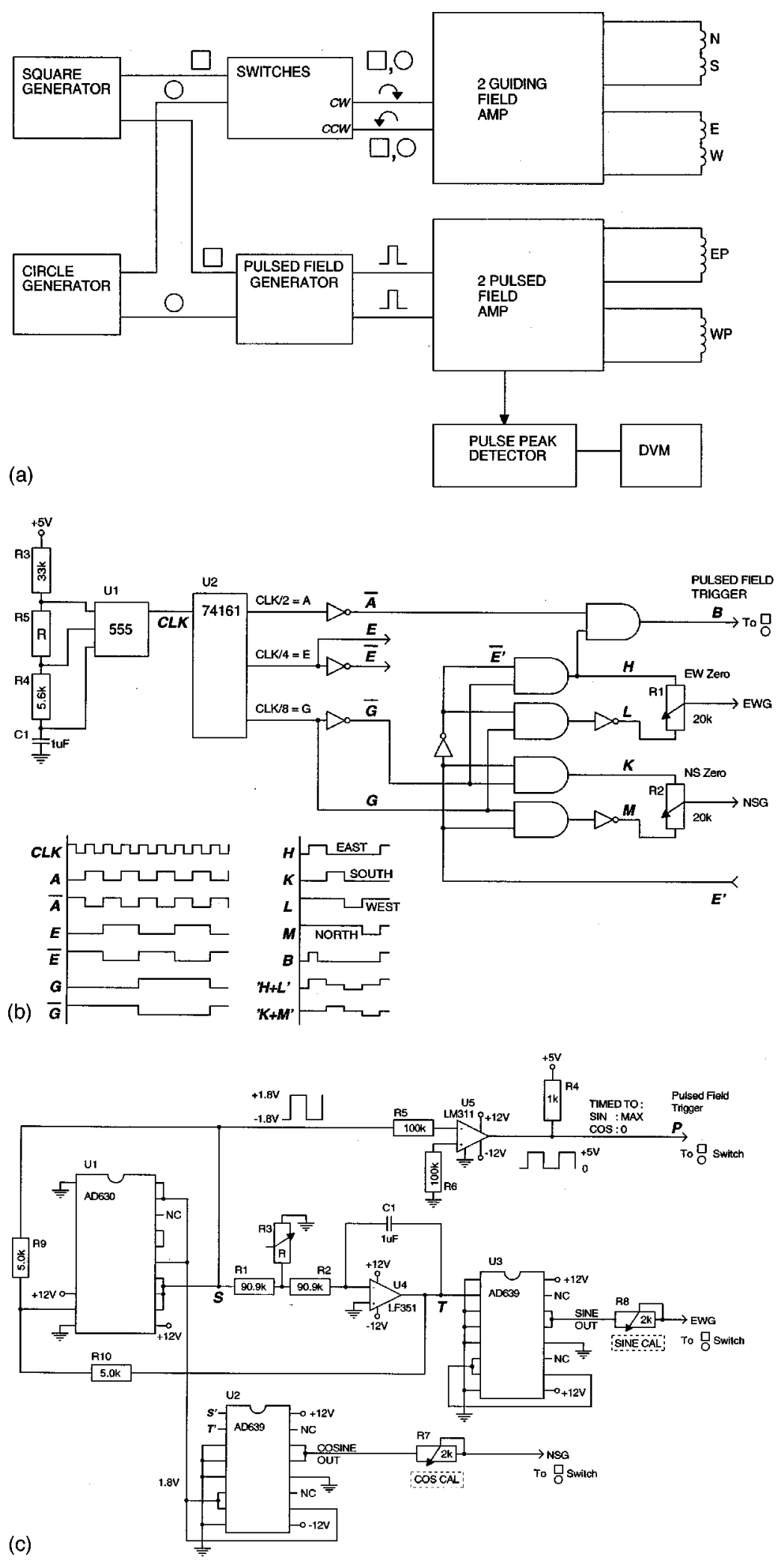

FIG. 3. (a) Block diagram of the magnetometer controls. Circuits, (b), (c), and (e) are used to generate the guiding field in the sample. There are two modes, square and circular, and two senses, $\mathrm{CW}$ and $\mathrm{CCW}$. Switches shown in Fig. 2(b) select the mode and the sense. Outputs from the square and circular generators are also used to control the timing in the pulsed-field generator, circuit d. (b) Square generator. Outputs go to the two guiding-field amplifiers when the square mode is selected at the square/circular mode switch [see front panel, Fig. 2(b)]. The sense is selected by the CW/CCW switch. The switch connections are indicated in Sec. III G. (c) Circle generator. When the circular mode is selected, the outputs EWG and NSG are connected through the square/circle mode switch to the second stage of the guiding-field amplifiers (e) at the inputs EW' $^{\prime}$ and NS' and switch $S_{1}$ is open. The sense of rotation is selected by the $\mathrm{CW} / \mathrm{CCW}$ switch. The switch connections are given in Sec. III G and are selected by the SENSE switch in Fig. 2(b). (d) Pulsed-field generator. Input from either circuit (b) or (c) (depending on the mode switch) enters at Point N. The outputs EP and WP go to the two pulsed-field amplifiers (f). (e) Guiding-field amplifiers. Input is from the square generator [circuit (b)] at $R_{1}$ or from the circle generator circuit (c) at $R_{9}$, depending on the mode switch selection [Fig. 2(b)], which is represented by the switches $S_{1}$ and $S_{2}$. For the square mode, $S_{1}$ is closed and $S_{2}$ is open; for the circular mode $S_{1}$ is open and $S_{2}$ is closed. (f) Pulsed-field amplifiers. $4 \mathrm{~V}, 1-5$-ms-wide pulses (EP, WP) from circuit (d) generate current pulses in the coils EP and WP. (g) Pulse-peak detector.

\section{ELECTRONIC CIRCUITS AND THEIR FUNCTIONS}

A comprehensive block diagram of the subcircuits of the magnetometer is given in Fig. 3(a). The circuits indicated are discussed separately below.

\section{A. "Square" generator [Fig. 3(b)]}

This circuit is called the square generator because it produces a stepped magnetic-field sequence that causes motile magnetotactic bacteria to swim in a (horizontal) square. It starts from the classic No. 555 timer chip that delivers a +5 $\mathrm{V}$ square wave with a variable frequency that can be set by a switch that selects one of four different fixed resistors $(R$ $=32.2,80,127$, and $375 \mathrm{~K}$ ) or one variable resistor of $1 \mathrm{M} \Omega$. From this clock signal other square waves are derived by dividing the frequency by 2,4 , or 8 in a No. 74161 chip 

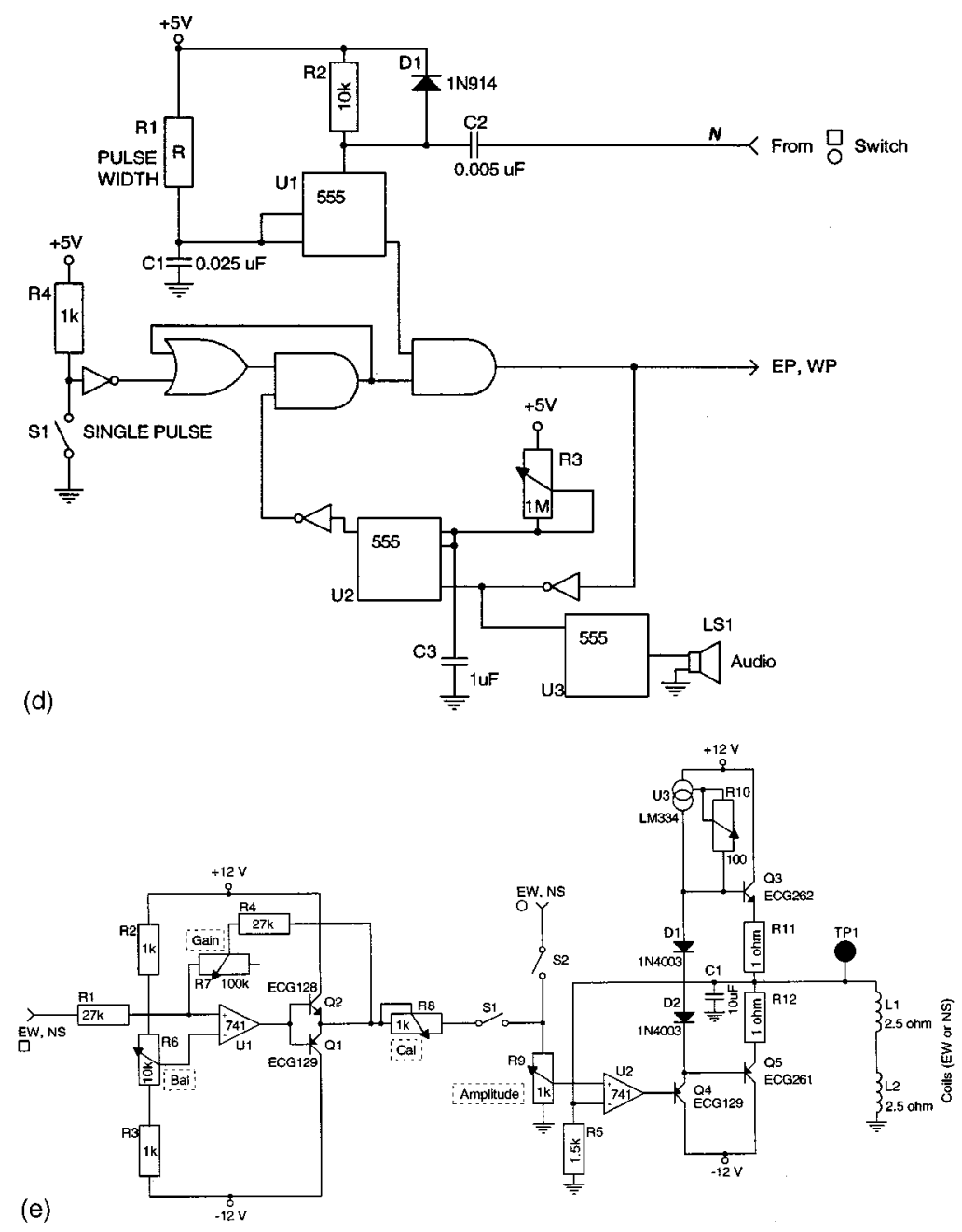

FIG. 3. (Continued.)

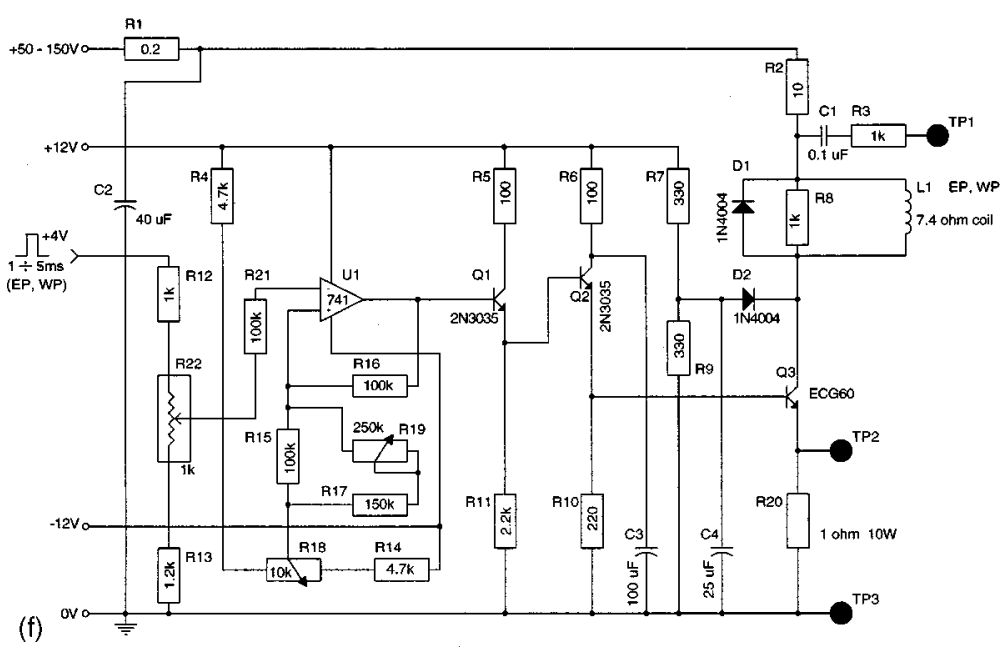

(designated by $\mathbf{A}, \overline{\mathbf{A}}, \mathbf{E}, \overline{\mathbf{E}}$, and $\mathbf{G}, \overline{\mathbf{G}}$, respectively). They are composed by adders and AND gates to the signals $\mathbf{H}, \mathbf{L}, \mathbf{K}$, and $\mathbf{M}$ and then to the trapezoidal shape of the signals for the guiding fields $\mathbf{H}+\mathbf{L}, \mathbf{K}+\mathbf{M}$ in the pulse-shape diagram and north-south gate (NSG) and east-west gate (EWG) at the outputs. The bacteria swim in a square provided the relative amplitudes of the component currents are properly adjusted, which is part of the calibration procedure. This path is generated for each period $T$ of the guiding field if the currents through the coils produce a field $B_{0}$ at the sample in the following sequence:

$$
\begin{aligned}
& 0 \rightarrow T / 4, \quad B(\mathrm{NS})=+B_{0}, \quad B(\mathrm{EW})=0, \\
& T / 4 \rightarrow T / 2, \quad B(\mathrm{EW})=+B_{0}, \quad B(\mathrm{NS})=0, \\
& T / 2 \rightarrow 3 T / 4, \quad B(\mathrm{NS})=-B_{0}, \quad B(\mathrm{EW})=0, \\
& 3 T / 4 \rightarrow T, \quad B(\mathrm{EW})=-B_{0}, \quad B(\mathrm{NS})=0 .
\end{aligned}
$$




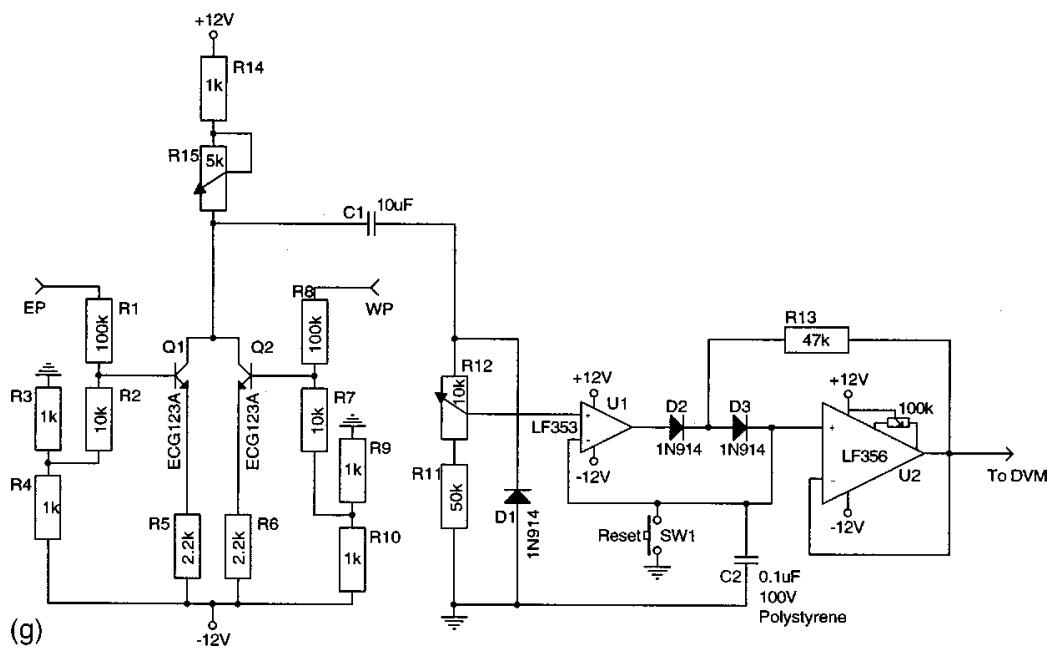

FIG. 3. (Continued.)

\section{B. “Circle” generator [Fig. 3(c)]}

Sinusoidal currents producing a magnetic field of amplitude $B_{0}$ in the NS and EW coils achieve the rotating guiding field for circular bacterial motion:

$B(\mathrm{NS})=B_{0} \cos (\omega t), \quad B(\mathrm{EW})=B_{0} \sin (\omega t), \quad(T=2 \pi / \omega)$.

These wave forms are simultaneously realized with a quadrature oscillator, such as is available from Analog Devices. ${ }^{11}$ This device contains chips of types AD630, 2xAD639, and a LF351 (used as an integrator) [Fig. 3(c)]. The period $T$ is determined by the value of the resistor $R$ that can be switched to $22 \mathrm{~K}, 8.1 \mathrm{~K}, 2.8 \mathrm{~K}$, or $584 \Omega$, corresponding to $2,4,8,16 \mathrm{~s}$, respectively, or to a $1 \mathrm{M} \Omega$ variable resistor. The output signals EWG and NSG are connected to a switch in circuit (g) (Fig. 3) that selects the square or the circular mode. A trigger pulse is also generated in circuit (c) (Fig. 3) output $\mathrm{P}$ for the pulsed-field generation in unit (e) (Fig. 3) when the system is in the circular mode.

\section{Pulsed-field generator [Fig. 3(d)]}

This circuit combines signals from various sources to yield input pulses at the right time and duration for the pulse amplifiers, but only once after the "single-pulse" switch is closed. Depending on the guiding-field mode (square/circle), this lets through one pulse that comes from a trigger at point $\mathrm{N}$, resulting either from point $\mathrm{B}$ on the square generator [Fig. 3 (b)] or from point $\mathrm{P}$ on the circle generator [Fig. 3(c)]. Both of these pulses are timed to occur at the middle of a leg in the square mode, or at a quarter of a full circle in the circular mode. The triggers are then transformed to clean, rectangular pulses by the 555 timer U1, with a width determined by the value of resistor $R_{1}$; for $R_{1}=30,83,127,169$, and $211 \mathrm{~K}$ ), the pulse width is $1,2,3,4$, and $5 \mathrm{~ms}$, respectively.

\section{Guiding-field amplifiers [Fig. 3(e)]}

The guiding-field amplitude can be varied from $0 \mathrm{G}$ up to about $15 \mathrm{G}$ with $R_{9}$. The maximum value of the guiding field, $15 \mathrm{G}$, exceeds the geomagnetic field by about a factor of 30, large enough to neglect the geomagnetic field in most cases, but not so large that it would cause excessive heating of the coils or of the circuit itself. The square or circular shape of the guiding fields is prescribed by signals from the pulse logic, as discussed in Secs. III A and III B. The duration of one cycle can be set from 2 to $16 \mathrm{~s}$ in four steps of 2 by the switched resistor $R_{5}$ in Fig. 3(b), or continuously.

The preamplifiers consist of 741 opamps followed by bipolar followers. These provide a voltage gain of about 1 and deliver a balanced signal to the ECG 261/262 heat-sinkprotected power amplifiers that feed the NS and EW guidingfield coil pairs. The balance (Bal) and the zero-field setting (Gain) of the coils are adjusted with trimpots $R_{6}$ and $R_{7}$, as indicated in the circuit diagram.

\section{E. Pulsed-field amplifiers [Fig. 3(f)]}

There are two identical power amplifiers, one for each coil, that generate the pulsed-field current. The pulse fields, $B_{p}(E)$ and $B_{p}(W)$, are normally equal. The reason for having separate amplifiers is that for the available coil size, the voltage or current would be excessive if the coils were placed in series or parallel, respectively. With the present arrangement, the maximum field at the sample position is $800 \mathrm{G}$ for $+4 \mathrm{~V}$ (standard) pulses at the inputs of both amplifiers. The input signals pass first through $1 \mathrm{k} \Omega$ thumb wheel attenuators $R_{22}$ (Hartmann Gerätebau, Beiersdorf, Germany, 20030) that allow the pulsed-field amplitude to be varied in small steps [Fig. 2(b), right]. Next, the attenuated pulses are applied to opamps (741) where the quiescent dc current in the power stage (ECG60) is set with the aid of the 741 bias (the $10 \mathrm{~K}$ pot $R_{18}$ ) at a value about $+1 \mathrm{mV}$ between test points TP2 and TP3 [EP ZERO and WP ZERO in Fig. 2(b)].

The input signal at the base of the ECG60 is delivered through two, cascaded, emitter followers (2N3035). To prevent bottoming of the ECG60, a diode $D_{2}$ is connected from the collector of the ECG60 to a point clamped at $+6 \mathrm{~V}$ during the pulse. The maximum output pulse is measured as a voltage across the $10 \Omega$ resistors placed in series with the EP and WP coils. The oscilloscope test point TP1 for the pulses is provided at the bottom of the $10 \Omega$ resistor $R_{2}$ placed in series with the coil. A diode + resistor clamp $D_{1} R_{8}$ prevents the pulse coil from producing an inductive overshoot after each pulse. 


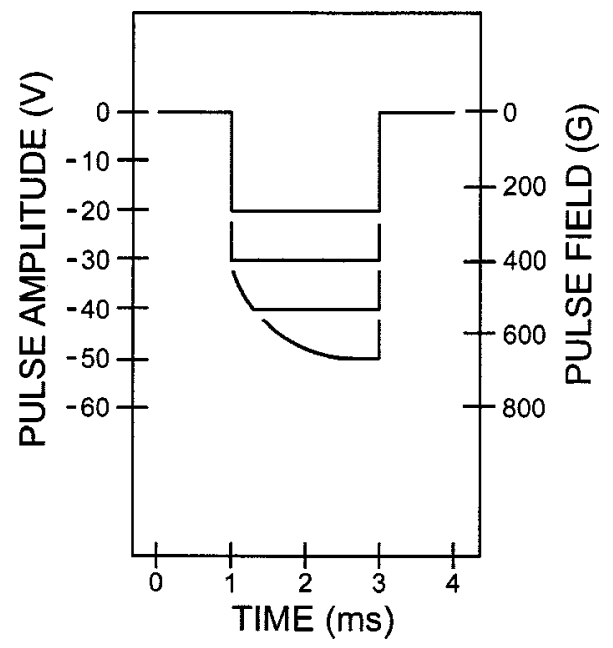

FIG. 4. Pulsed-field shape from the pulse amplifiers (see Sec. III E). The pulses become distorted above $400 \mathrm{G}$, but a unique maximum value is determined by the pulse-peak detector [Fig. 3(g)].

The pulse current supply must deliver a voltage of +50 to $+150 \mathrm{~V}$ with respect to ground and be resistant to the $\sim 5$ ms 10 A pulses, but it does not need much long-term stability. The two other supplies $(+12$ and $-12 \mathrm{~V}$ with respect to ground) need not deliver more than about $200 \mathrm{~mA}$, and modest regulation is sufficient.

\section{F. Pulse peak detector [Fig. 3(g)]}

The pulse field is not very linear as a function of the setting of the pulse amplitude control $R_{22}$ in Fig. 3(f), and, moreover, the top of the pulse is not flat at high pulse currents. The effective pulse duration is further reduced by the delaying effect of the inductance of the coil. This effect is shown in Fig. 4. Therefore, the maximum pulsed-field amplitude is measured with a pulse-peak detector. This circuit comprises two actions; the first stage is a simple adder that delivers the sum of the negative pulses appearing at the outputs of the pulsed-field amplifiers as applied to the $7.4 \Omega$ pulsed-field coils, and the second stage is the detector proper. ${ }^{12}$ The feedback in this circuit very effectively clamps the output voltage to the top value of the output pulse from the adder. This value is read with a digital voltmeter (DVM) between the test point DVM and ground after each pulse.

\section{G. Switches}

This part of the circuit only contains switches between parts of other circuits; the connections are summarized as follows:

\section{Square guiding field}

Circuit (b) output (EW) $\rightarrow$ circuit (e) input (EW $\square) ; S_{1}$ closed, $S_{2}$ open.

Circuit (b) output (NS) $\rightarrow$ circuit (e) input (NS $\square$ ); $S_{1}$ closed, $S_{2}$ open.

\section{Circle guiding field}

Circuit (c) output (EWG) $\rightarrow$ circuit (e) input $(E W \bigcirc) ; S_{1}$ open, $S_{2}$ closed.

Circuit (c) output (NSG) $\rightarrow$ circuit (e) input (NS $\bigcirc$ ); $S_{1}$ open, $S_{2}$ closed.

\section{Switching between clockwise (CW) and counter- clockwise (CCW) motion}

Circuit (b) CW: output (E) $\rightarrow$ input $\left(\mathbf{E}^{\prime}\right)$; CCW: output $(\overline{\mathbf{E}}) \rightarrow$ input $\left(\mathbf{E}^{\prime}\right)$.

Circuit (b) CW: point $\mathbf{S} \rightarrow$ point $\mathbf{S}^{\prime}$; point $\mathbf{T} \rightarrow$ point $\mathbf{T}^{\prime}$; CCW: point $\mathbf{S} \rightarrow$ point $\mathbf{T}^{\prime}$; point $\mathbf{T} \rightarrow$ point $\mathbf{S}^{\prime}$.

\section{ACKNOWLEDGMENTS}

The authors wish to thank Jerry T. Ryle for CAD conversion of most raw drawings into first-class diagrams. One of the authors (R.B.F.) acknowledges support from the National Science Foundation.

${ }^{1}$ R. P. Blakemore, Science 190, 377 (1975).

${ }^{2}$ D. A. Bazylinski, Am. Soc. Microbiol. News 61, 337 (1995).

${ }^{3}$ T. Sakaguchi, J. G. Burgess, and T. Matsunaga, Nature (London) 365, 47 (1993).

${ }^{4}$ N. Petersen, T. von Dobeneck, and H. Vali, Nature (London) 320, 611 (1986).

${ }^{5}$ R. E. Dunin-Borkowski, M. R. McCartney, R. B. Frankel, D. A. Bazylinski, M. Pósfai, and P. R. Buseck, Science 282, 1868 (1998).

${ }^{6}$ R. B. Frankel, Annu. Rev. Biophys. Bioeng. 13, 85 (1984).

${ }^{7}$ J. E. Knowles, IEEE Trans. Magn. 14, 858 (1978).

${ }^{8}$ I. Penninga, H. de Waard, B. M. Moskowitz, D. A. Bazylinski, and R. B. Frankel, J. Magn. Magn. Mater. 149, 279 (1995).

${ }^{9}$ N. Petersen, D. G. Weiss, and H. Vali, in Geomagnetism and Paleomagnetism, edited by F. J. Lowes, D. W. Collision, J. H. Parry, S. K. Runcorn, D. C. Tozer, and A. Soward (Kluwer, Dordrecht, Amsterdam, 1989), pp. 231-241.

${ }^{10}$ A. Iadicicco and C. P. Bean (unpublished).

${ }^{11}$ Analog Devices, One Technology Way, P.O. Box 9106, Norwood, MA 02062-9106.

${ }^{12} \mathrm{P}$. Horowitz and W. Hill, The Art of Electronics, 2nd ed. (Cambridge University, Cambridge, UK, 1989), p. 217. 